\title{
Multiple solutions for asymptotically linear elliptic equations with sign-changing weight
}

\author{
Nikolaos S. Papageorgiou and Vicenţiu D. Rădulescu
}

\begin{abstract}
We consider a semilinear Dirichlet problem driven by the Laplacian and with an indefinite (that is, sign-changing) weight and a nonlinearity which is asymptotically linear near $\pm \infty$. Using variational methods together with truncation techniques and Morse theory, we show that the problem has at least three nontrivial solutions, two of which have constant sign (one positive and the other negative).
\end{abstract}

\section{Introduction}

Let $\Omega \subseteq \mathbb{R}^{N}$ be a bounded domain with a $C^{2}$-boundary $\partial \Omega$. In this paper, we study the existence of multiple nontrivial solutions for the following semilinear Dirichlet problem:

$$
-\Delta u(z)=\beta(z) f(u(z)) \quad \text { in } \Omega,\left.\quad u\right|_{\partial \Omega}=0 .
$$

In this problem the weight function $\beta \in L^{\infty}(\Omega)$ is nodal (that is, sign changing) and $f$ is a $C^{1}$-nonlinearity which exhibits linear growth near $\pm \infty$ and is superlinear near 0 . Using variational methods coupled with suitable truncation and comparison techniques and Morse theory (critical groups), we show that (1) has at least three nontrivial solutions, two of which have constant sign (one positive and the other negative).

Problems with an indefinite nonlinearity were first investigated by Ouyang [11] on a compact Riemannian manifold using bifurcation theory. Subsequently, Alama and Tarantello [1], [2] using variational methods extended the results of Ouyang [11] by considering more general nonlinearities and assuming a thickness condition of the form $\bar{\Omega}_{+} \cap \bar{\Omega}_{-}=\emptyset$, where $\Omega_{+}=\{z \in \Omega: \beta(z)>0\}$ and $\Omega_{-}=\{z \in$ $\Omega: \beta(z)<0\}$. This condition was removed by Berestycki, Capuzzo-Dolcetta, and Nirenberg [7], who proved the existence of solutions by deriving a priori bounds and using topological methods. However, their condition on the weight $\beta(\cdot)$ is stronger since $\beta \in C^{1}(\Omega)$, and they assume a nondegeneracy condition of the form $\nabla \beta(z) \neq 0$ when $\beta(z)=0$. (That is, the level set $[\beta=0]$ is a $C^{1}$-submanifold; so, in this case the zero set is thin.) Three-solutions theorems for problems with

Kyoto Journal of Mathematics, Vol. 55, No. 3 (2015), 593-606

DOI $10.1215 / 21562261-3089082$, (C) 2015 by Kyoto University

Received February 12, 2014. Revised June 30, 2014. Accepted July 3, 2014.

2010 Mathematics Subject Classification: Primary 35J20; Secondary 35J60, 58E05. 
a superlinear $f(\cdot)$ satisfying the Ambrosetti-Rabinowitz condition, without any thickness or thinness condition, were proved for parametric problems and for certain values of the parameter, by Chang and Jiang [8].

To the best of our knowledge, the first paper involving nonlinear elliptic equations with asymptotically linear terms $f(z, u)$ is due to Amann and Zehnder [3], in the context of semilinear equations (i.e., if $p=2$ ). They proved an exis-

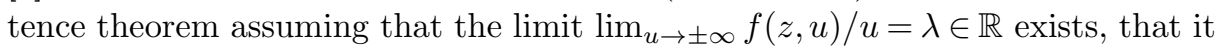
does not belong to the spectrum of the Laplace operator in $H_{0}^{1}(\Omega)$ (nonresonance at infinity), and that there is at least one eigenvalue between $\lambda$ and $\lambda+f^{\prime}(0)$. Related multiplicity results for Dirichlet elliptic problems involving asymptotically linear terms near $\pm \infty$ have been studied in some recent papers (see $\mathrm{Hu}$ and Papageorgiou [10] and Papageorgiou and Smyrlis [14]). The hypotheses in the present paper are more general than those imposed in [10] and [14]. For instance, $\mathrm{Hu}$ and Papageorgiou [10] establish a three-nontrivial-solutions theorem provided that $f$ satisfies a local boundedness assumption and $f_{u}(z, u)$ has $q$-polynomial growth for some $0<q<4 /(N-2)$. Papageorgiou and Smyrlis [14] produce five nontrivial smooth solutions, two positive, two negative, and one nodal. This is done under several hypotheses, including the behavior of the quotient $f(z, u) /\left(|u|^{p-2} u\right)$ with respect to the first two eigenvalues of the $p$-Laplace operator, the existence of two zeros of the nonlinear term, and a certain monotonicity assumption with respect to the mapping $u \mapsto f(z, u)+|u|^{p-2} u$ (see [14, pp. 3138, 3142]). With respect to these works, the main features of the present paper are the following: (1) the presence of a sign-changing weight; and (2) the study is performed for nonlinearities that fulfill general assumptions and whose behavior is described with respect to certain eigenvalues of a weighted eigenvalue problem that involves the indefinite potential.

Throughout this paper, for all $x \in \mathbb{R}$, we denote $x^{ \pm}=\max \{ \pm x, 0\}$.

\section{Mathematical background}

In the analysis of problem (1), we will use the Sobolev space $H_{0}^{1}(\Omega)$ and the Banach space $C_{0}^{1}(\bar{\Omega})=\left\{u \in C^{1}(\bar{\Omega}):\left.u\right|_{\partial \Omega=0}\right\}$. We know that $C_{0}^{1}(\bar{\Omega})$ is an ordered Banach space with positive cone

$$
C_{+}(\bar{\Omega})=\left\{u \in C_{0}^{1}(\bar{\Omega}): u(z) \geq 0 \text { for all } z \in \bar{\Omega}\right\} .
$$

This cone has a nonempty interior given by

$$
\operatorname{int} C_{+}(\bar{\Omega})=\left\{u \in C_{+}(\bar{\Omega}): u(z)>0 \text { for all } z \in \Omega,\left.\frac{\partial u}{\partial n}\right|_{\partial \Omega}<0\right\}
$$

with $n(\cdot)$ being the outward unit normal on $\partial \Omega$. Also, prominent in our arguments will be the spectrum of the following weighted linear eigenvalue problem:

$$
-\Delta u(z)=\lambda \beta(z) u(z) \quad \text { in } \Omega,\left.\quad u\right|_{\partial \Omega}=0 .
$$

When the weight $\beta \in L^{\infty}(\Omega)$ is nodal, it is well known (see, e.g., Gasinski and Papageorgiou [9, p. 714]) that problem (2) has a double sequence of distinct 
eigenvalues

$$
\cdots<\hat{\lambda}_{k}^{-}(\beta)<\cdots<\hat{\lambda}_{2}^{-}(\beta)<\hat{\lambda}_{1}^{-}(\beta)<0<\hat{\lambda}_{1}^{+}(\beta)<\hat{\lambda}_{2}^{+}(\beta)<\cdots<\hat{\lambda}_{k}^{+}(\beta)<\cdots .
$$

Let $|\cdot|_{N}$ denote the Lebesgue measure on $\mathbb{R}^{N}$. If $\Omega_{+}=\{z \in \Omega: \beta(z)>0\}$ and $\left|\Omega_{+}\right|_{N}>0$, then $\hat{\lambda}_{n}^{+}(\beta) \rightarrow+\infty$ as $n \rightarrow \infty$. Similarly, if $\Omega_{-}=\{z \in \Omega: \beta(z)<$ $0\}$ and $\left|\Omega_{-}\right|_{N}>0$, then $\hat{\lambda}_{n}^{-}(\beta) \rightarrow-\infty$. On the other hand, if $\left|\Omega_{+}\right|_{N}=0$, then $\hat{\lambda}_{n}^{+}(\beta)=0$ for all $n \geq 1$, while if $\left|\Omega_{-}\right|_{N}=0$, then $\hat{\lambda}_{n}^{-}(\beta)=0$ for all $n \geq 1$.

Suppose that $\Omega_{+}$is an open connected set with a $C^{2}$-boundary $\partial \Omega_{+}$, and consider the following weighted linear eigenvalue problem:

$$
-\Delta u(z)=\lambda \beta^{+}(z) u(z) \text { in } \Omega_{+},\left.\quad u\right|_{\partial \Omega_{+}}=0 .
$$

According to our previous discussion, problem (3) has only positive eigenvalues, namely, $\left\{\hat{\lambda}_{k}^{\Omega_{+}}\left(\beta^{+}\right)\right\}_{k \geq 1}, \hat{\lambda}_{k}^{\Omega_{+}}\left(\beta^{+}\right) \rightarrow+\infty$ as $k \rightarrow \infty$, and $\hat{\lambda}_{1}^{\Omega_{+}}\left(\beta^{+}\right)>0$, and it is simple. We have the following variational characterization of $\hat{\lambda}_{1}^{\Omega_{+}}\left(\beta^{+}\right)$:

$$
\hat{\lambda}_{1}^{\Omega_{+}}\left(\beta^{+}\right)=\inf \left[\frac{\|D u\|_{L^{2}\left(\Omega_{+}, \mathbb{R}^{N}\right)}^{2}}{\int_{\Omega_{+}} \beta^{+}(z) u^{2} d z}: u \in H_{0}^{1}\left(\Omega_{+}\right), u \neq 0\right] .
$$

The infimum in relation (4) is realized on the one-dimensional eigenspace corresponding to $\hat{\lambda}_{1}^{\Omega_{+}}\left(\beta^{+}\right)>0$. Standard regularity theory implies that the elements of this eigenspace belong in $C_{0}^{1}\left(\overline{\Omega_{+}}\right)$. In fact from (4) we can see that they do not change sign. In what follows by $\tilde{u}_{1}\left(\Omega_{+}\right)$we denote the $L^{2}$-normalized (i.e., $\left.\left\|\tilde{u}_{1}\left(\Omega_{+}\right)\right\|_{L^{2}\left(\Omega_{+}\right)}=1\right)$ positive eigenfunction. Using the maximum principle (see, e.g., Gasinski and Papageorgiou [9, p. 738]) we have $\tilde{u}_{1}\left(\Omega_{+}\right) \in \operatorname{int} C_{+}\left(\Omega_{+}\right)$.

Our variational approach will be based on the well-known mountain pass theorem of Ambrosetti and Rabinowitz [5], formulated here in a slightly more general form using the $C$-compactness condition on the functional instead of the more common $P S$-condition (Palais-Smale condition; see [9, Theorem 5.2.5]).

So, let $X$ be Banach, and let $X^{*}$ be its topological dual. By $\langle\cdot, \cdot\rangle$ we denote the duality brackets for the pair $\left(X^{*}, X\right)$. Given $\varphi \in C^{1}(X)$, we say that $\varphi$ satisfies the $C$-condition, if the following is true:

Every sequence $\left\{u_{n}\right\}_{n \geq 1} \subseteq X$ such that $\left\{\varphi\left(u_{n}\right)\right\}_{n \geq 1} \subseteq \mathbb{R}$ is bounded and

$$
\left(1+\left\|u_{n}\right\|\right) \varphi^{\prime}\left(u_{n}\right) \rightarrow 0 \quad \text { in } X^{*} \text { as } n \rightarrow \infty
$$

admits a strongly convergent subsequence.

This compactness-type condition on $\varphi$ leads to a deformation theorem, which in turn produces a minimax theory for the critical values of $\varphi$. A major result in that theory is the mountain pass theorem.

\section{THEOREM 1}

Assume that $\varphi \in C^{1}(X)$ satisfies the $C$-condition, and assume that $u_{0}, u_{1} \in X$, $\rho>0,\left\|u_{1}-u_{0}\right\|>\rho$,

$$
\max \left\{\varphi\left(u_{0}\right), \varphi\left(u_{1}\right)\right\}<\inf \left[\varphi(u):\left\|u-u_{0}\right\|=\rho\right]=m_{\rho},
$$


and $c=\inf _{\gamma \in \Gamma} \max _{0 \leq t \leq 1} \varphi(\gamma(t))$, where $\Gamma=\left\{\gamma \in C([0,1], X): \gamma(0)=u_{0}, \gamma(1)=\right.$ $\left.u_{1}\right\}$. Then $c \geq m_{\rho}$ and $c$ is a critical value of $\varphi$.

Also, we will use some tools from Morse theory (critical groups), which for the benefit of the reader we briefly review below. So, for $\varphi \in C^{1}(X)$ and $c \in \mathbb{R}$, we introduce the following sets:

$$
\begin{aligned}
\varphi^{c} & =\{u \in X: \varphi(u) \leq c\}, \quad K_{\varphi}=\left\{u \in X: \varphi^{\prime}(u)=0\right\}, \quad \text { and } \\
K_{\varphi}^{c} & =\left\{u \in K_{\varphi}: \varphi(u)=c\right\} .
\end{aligned}
$$

Let $\left(Y_{1}, Y_{2}\right)$ be a topological pair such that $Y_{2} \subseteq Y_{1} \subseteq X$. For every integer $k \geq 0$ by $H_{k}\left(Y_{1}, Y_{2}\right)$ we denote the $k$ th relative singular homology group with integer coefficients for the pair $\left(Y_{1}, Y_{2}\right)$. The critical groups of $\varphi$ at an isolated $u \in K_{\varphi}^{c}$ are defined by

$$
C_{k}(\varphi, u)=H_{k}\left(\varphi^{c} \cap U, \varphi^{c} \cap U \backslash\{u\}\right) \quad \text { for all } k \geq 0,
$$

where $U$ is a neighborhood of $u$ such that $K_{\varphi} \cap \varphi^{c} \cap U=\{u\}$. The excision property of singular homology theory implies that this definition is independent of the particular choice of the neighborhood $U$.

Suppose that $\varphi \in C^{1}(X)$ satisfies the $C$-condition and $\inf \varphi\left(K_{\varphi}\right)>-\infty$. Let $c<\inf \varphi\left(K_{\varphi}\right)$. The critical groups of $\varphi$ at infinity are defined by

$$
C_{k}(\varphi, \infty)=H_{k}\left(X, \varphi^{c}\right) \text { for all } k \geq 0 .
$$

Using the second deformation theorem (see, e.g., Gasinski and Papageorgiou [9, p. 628]), we see that this definition is independent of the particular choice of the level $c<\inf \varphi\left(K_{\varphi}\right)$. We know that if $C_{k}(\varphi, \infty) \neq 0$, then there exists $u \in K_{\varphi}$ such that $C_{k}(\varphi, u) \neq 0$ (a consequence of the so-called Morse relation; see, e.g., Ambrosetti and Malchiodi [4, p. 222]).

Recently the authors proved the following result, which is useful in the computation of critical groups at infinity (see Papageorgiou and Rădulescu [13]).

\section{PROPOSITION 2}

Assume that $(t, u) \mapsto h_{t}(u)$ belongs to $C^{1}([0,1] \times X)$ and maps bounded sets to bounded sets, that the maps $u \rightarrow\left(h_{t}\right)^{\prime}(u)$ and $t \mapsto \partial_{t} h_{t}(u)$ are both locally Lipschitz, that $h_{0}$ and $h_{1}$ satisfy the $C$-condition,

$$
\left|\partial_{t} h_{t}(u)\right| \leq c_{1}\|u\|^{p} \quad \text { for all } u \in X,
$$

with $c_{1}>0,1<p<\infty$, and that there exist $\xi_{0} \in \mathbb{R}$ and $\delta_{0}>0$ such that

$$
h_{t}(u) \leq \xi_{0} \quad \Rightarrow \quad(1+\|u\|)\left\|\left(h_{t}\right)^{\prime}(u)\right\|_{*} \geq \delta_{0}\|u\|^{p} \quad \text { for all } t \in[0,1] .
$$

Then $C_{k}\left(h_{0}, \infty\right)=C_{k}\left(h_{1}, \infty\right)$ for all $k \geq 0$.

We conclude this section by fixing our notation. In what follows by $\|\cdot\|$ we denote the norm of the Sobolev space $H_{0}^{1}(\Omega)$. By virtue of the Poincaré inequality, we 
have that

$$
\|u\|=\|D u\|_{L^{2}\left(\Omega, \mathbb{R}^{N}\right)} \quad \text { for all } u \in H_{0}^{1}(\Omega) .
$$

For all $u \in H_{0}^{1}(\Omega)$, we define $u^{ \pm}(\cdot)=u(\cdot)^{ \pm}$. We know that

$$
u^{ \pm} \in H_{0}^{1}(\Omega), \quad u=u^{+}-u^{-}, \quad|u|=u^{+}+u^{-} .
$$

Also, by $N_{f}$ we denote the Nemitsky (superposition) operator corresponding to $f$, that is,

$$
N_{f}(u)(\cdot)=f(u(\cdot)) \quad \text { for all } u \in H_{0}^{1}(\Omega) .
$$

Finally by $A \in \mathcal{L}\left(H_{0}^{1}(\Omega), H^{-1}(\Omega)=H_{0}^{1}(\Omega)^{*}\right)$ we denote the bounded linear operator defined by

$$
\langle A(u), y\rangle=\int_{\Omega}(D u, D y)_{\mathbb{R}^{N}} d z \quad \text { for all } u, y \in H_{0}^{1}(\Omega) .
$$

\section{Multiplicity theorem}

Our hypotheses on the data of problem (1) are the following.

$H(\beta): \beta \in L^{\infty}(\Omega), \beta^{+}, \beta^{-} \neq 0$, and $\Omega_{+}=\{z \in \Omega: \beta(z)>0\}$ is a connected open set with a $C^{2}$-boundary $\partial \Omega_{+}$.

$H(f): f \in C^{1}(\mathbb{R}), f(0)=0$, and

(i) $\left|f^{\prime}(x)\right| \leq a\left(1+|x|^{r-1}\right)$ for all $x \in \mathbb{R}$ with $a>0$ and

$$
2 \leq r<2^{*}= \begin{cases}\frac{2 N}{N-2} & \text { if } N \geq 3 \\ +\infty & \text { if } N=1,2\end{cases}
$$

(ii) there exists an integer $m \geq 2$ such that

$$
\begin{aligned}
\hat{\lambda}_{1}^{\Omega_{+}}\left(\beta^{+}\right) & <\hat{\lambda}_{m}^{+}(\beta), \\
\hat{\lambda}_{m}^{+}(\beta) & <\liminf _{x \rightarrow \pm \infty} \frac{f(x)}{x} \leq \limsup _{x \rightarrow \pm \infty} \frac{f(x)}{x}<\hat{\lambda}_{m+1}^{+}(\beta) ;
\end{aligned}
$$

(iii) $f^{\prime}(x)=\lim _{x \rightarrow 0} f(x) / x=0$.

First we will produce two constant-sign solutions, one positive and the other negative. To this end, we introduce the positive and negative truncations of $f(\cdot)$, namely, the $C^{1}$-functions (see hypothesis $H(f)($ iii))

$$
f_{+}(x)=f\left(x^{+}\right) \quad \text { and } \quad f_{-}(x)=f\left(-x^{-}\right) \quad \text { for all } x \in \mathbb{R} .
$$

Let $F_{ \pm}(x)=\int_{0}^{x} f_{ \pm}(s) d s$, and consider the $C^{2}$-functionals $\varphi_{ \pm}: H_{0}^{1}(\Omega) \rightarrow \mathbb{R}$ defined by

$$
\varphi_{ \pm}(u)=\frac{1}{2}\|D u\|_{2}^{2}-\int_{\Omega} \beta(z) F_{ \pm}(u(z)) d z \quad \text { for all } u \in H_{0}^{1}(\Omega) .
$$

Also, let $\varphi: H_{0}^{1}(\Omega) \rightarrow \mathbb{R}$ be the energy functional for problem (1) defined by

$$
\varphi(u)=\frac{1}{2}\|D u\|_{2}^{2}-\int_{\Omega} \beta(z) F(u(z)) d z \quad \text { for all } u \in H_{0}^{1}(\Omega),
$$

where $F(x)=\int_{0}^{x} f(s) d s$. We know that $\varphi \in C^{2}\left(H_{0}^{1}(\Omega)\right)$. 
PROPOSITION 3

If hypotheses $H(\beta)$ and $H(f)$ hold, then the functionals $\varphi_{ \pm}$satisfy the $C$ condition.

Proof

We do the proof for the functional $\varphi_{+}$; the proof for $\varphi_{-}$is similar.

So, let $\left\{u_{n}\right\}_{n \geq 1} \subseteq H_{0}^{1}(\Omega)$ be a sequence such that $\left\{\varphi_{+}\left(u_{n}\right)\right\}_{n \geq 1} \subseteq \mathbb{R}$ is bounded and

$$
\left(1+\left\|u_{n}\right\|\right) \varphi_{+}^{\prime}\left(u_{n}\right) \rightarrow 0 \quad \text { in } H^{-1}(\Omega)=H_{0}^{1}(\Omega)^{*} .
$$

From (5), we have that

$$
\begin{aligned}
& \left|\left\langle A\left(u_{n}\right), h\right\rangle-\int_{\Omega} \beta(z) f_{+}\left(u_{n}\right) h d z\right| \leq \frac{\varepsilon_{n}\|h\|}{1+\left\|u_{n}\right\|} \\
& \quad \text { for all } h \in H_{0}^{1}(\Omega), \text { with } \varepsilon_{n} \rightarrow 0^{+} .
\end{aligned}
$$

In (6) we choose $h=-u_{n}^{-} \in H_{0}^{1}(\Omega)$. Then

$$
\begin{aligned}
& \left\|D u_{n}^{-}\right\|_{L^{2}\left(\Omega, \mathbb{R}^{N}\right)}^{2} \leq \varepsilon_{n} \quad \text { for all } n \geq 1 \\
& \Rightarrow \quad u_{n}^{-} \rightarrow 0 \quad \text { in } H_{0}^{1}(\Omega) .
\end{aligned}
$$

Suppose that $\left\|u_{n}^{+}\right\| \rightarrow \infty$. We set $y_{n}=u_{n}^{+} /\left\|u_{n}^{+}\right\|, n \geq 1$. Then $\left\|y_{n}\right\|=1, y_{n} \geq 0$ for all $n \geq 1$. So, by passing to a suitable subsequence if necessary, we may assume that

(8) $\quad y_{n} \stackrel{w}{\rightarrow} y \quad$ in $H_{0}^{1}(\Omega) \quad$ and $\quad y_{n} \rightarrow y \quad$ in $L^{2}(\Omega)$ as $n \rightarrow \infty$.

From (5) and (6) it follows that

$$
\left|\left\langle A\left(y_{n}\right), h\right\rangle-\int_{\Omega} \beta(z) \frac{f\left(u_{n}^{+}\right)}{\left\|u_{n}^{+}\right\|} h d z\right| \leq \varepsilon_{n}^{\prime}\|h\|
$$

$$
\text { for all } h \in H_{0}^{1}(\Omega) \text {, with } \varepsilon_{n}^{\prime} \rightarrow 0^{+} \text {as } n \rightarrow \infty \text {. }
$$

From hypotheses $H(\beta), H(f)(\mathrm{i})$, and $H(f)(\mathrm{ii})$, we deduce that $\left\{\beta N_{f}\left(u_{n}^{+}\right) /\right.$ $\left.\left\|u_{n}^{+}\right\|\right\}_{n \geq 1} \subseteq L^{2}(\Omega)$ is bounded. So, if in (9) we choose $h=y_{n}-y \in H_{0}^{1}(\Omega)$ and pass to the limit as $n \rightarrow \infty$, then using (8) we obtain that

$$
\begin{aligned}
& \lim _{n \rightarrow \infty}\left\langle A\left(y_{n}\right), y_{n}-y\right\rangle=0 \\
& \quad \Rightarrow \quad\left\|D y_{n}\right\|_{L^{2}\left(\Omega, \mathbb{R}^{N}\right)}^{2} \rightarrow\|D y\|_{L^{2}\left(\Omega, \mathbb{R}^{N}\right)}^{2} \\
& \Rightarrow \quad y_{n} \rightarrow y \quad \text { in } H_{0}^{1}(\Omega)
\end{aligned}
$$

(by the Kadec-Klee property of Hilbert spaces; see (8))

$$
\Rightarrow \quad\|y\|=1, \quad y \geq 0 .
$$

Since $\left\{\beta N_{f}\left(u_{n}^{+}\right) /\left\|u_{n}^{+}\right\|\right\}_{n \geq 1} \subseteq L^{2}(\Omega)$ is bounded, by passing to a suitable subsequence if necessary and using hypothesis $H(f)($ ii), we obtain that

$$
\beta \frac{N_{f}\left(u_{n}^{+}\right)}{\left\|u_{n}^{+}\right\|} \stackrel{w}{\rightarrow} \beta \eta y \quad \text { in } L^{2}(\Omega) \text { with } \eta \in\left(\hat{\lambda}_{m}^{+}(\beta), \hat{\lambda}_{m+1}^{+}(\beta)\right) .
$$


So, if in (9) we pass to the limit as $n \rightarrow \infty$ and use (10) and (12), then

$$
\begin{aligned}
& \langle A(y), h\rangle=\int_{\Omega} \beta(z) \eta y h d z \quad \text { for all } h \in H_{0}^{1}(\Omega) \\
& \Rightarrow \quad A(y)=\beta(z) \eta y \\
& \Rightarrow \quad-\Delta y(z)=\eta \beta(z) y(z) \quad \text { a.e. in } \Omega,\left.y\right|_{\partial \Omega}=0 .
\end{aligned}
$$

From (12) and (13) it follows that $y=0$, which contradicts (11). This implies that $\left\{u_{n}^{+}\right\}_{n \geq 1} \subseteq H_{0}^{1}(\Omega)$ is bounded. This in conjunction with (7) implies that $\left\{u_{n}\right\}_{n \geq 1} \subseteq H_{0}^{1}(\Omega)$ is bounded. So, we may assume that

$$
u_{n} \stackrel{w}{\rightarrow} u \quad \text { in } H_{0}^{1}(\Omega) \quad \text { and } \quad u_{n} \rightarrow u \quad \text { in } L^{2}(\Omega) .
$$

In (6) we choose $h=u_{n}-u \in H_{0}^{1}(\Omega)$, pass to the limit as $n \rightarrow \infty$, and use (6).

We have that

$$
\begin{aligned}
& \lim _{n \rightarrow \infty}\left\langle A\left(u_{n}\right), u_{n}-u\right\rangle=0 \\
& \Rightarrow u_{n} \rightarrow u \quad \text { in } H_{0}^{1}(\Omega) \text { (as before via the Kadec-Klee property) } \\
& \Rightarrow \quad \varphi_{+} \quad \text { satisfies the } C \text {-condition. }
\end{aligned}
$$

In a similar fashion, we show that $\varphi_{-}$satisfies the $C$-condition.

Minor changes in the above proof lead to the following result.

\section{PROPOSITION 4}

If hypotheses $H(\beta)$ and $H(f)$ hold, then the energy functional $\varphi$ satisfies the C-condition.

Next we show that the functionals $\varphi_{ \pm}$satisfy the mountain pass geometry.

\section{PROPOSITION 5}

If hypotheses $H(\beta)$ and $H(f)$ hold, then there exist $\rho_{ \pm}>0$ such that $\inf \left[\varphi_{ \pm}(u)\right.$ : $\left.\|u\|=\rho_{ \pm}\right]=\hat{m}_{ \pm}>0$.

\section{Proof}

We do the proof for the functional $\varphi_{+}$; the proof for $\varphi_{-}$is similar.

Hypothesis $H(f)$ implies that, given $\varepsilon>0$ and $\vartheta \in\left(2,2^{*}\right)$, we can find $c_{1}=$ $c_{1}(\varepsilon, \vartheta)>0$ such that

$$
\begin{aligned}
& |f(x)| \leq \varepsilon|x|+c_{1}|x|^{\vartheta-1} \quad \text { for all } x \in \mathbb{R} \\
& \quad \Rightarrow \quad|F(x)| \leq \frac{\varepsilon}{2} x^{2}+c_{2}|x|^{\vartheta} \quad \text { for all } x \in \mathbb{R} \text { and with } c_{2}=\frac{c_{1}}{\vartheta}>0 .
\end{aligned}
$$

Then for all $u \in H^{1}(\Omega)$, we have that

$$
\begin{aligned}
\varphi_{+}(u) & =\frac{1}{2}\|D u\|_{L^{2}\left(\Omega, \mathbb{R}^{N}\right)}^{2}-\int_{\Omega} \beta(z) F\left(u^{+}\right) d z \\
& \geq \frac{1}{2}\|u\|^{2}-\frac{\varepsilon}{2}\|\beta\|_{L^{\infty}(\Omega)}\left\|u^{+}\right\|_{L^{2}(\Omega)}^{2}-c_{2}\left\|u^{+}\right\|_{L^{\vartheta}(\Omega)}^{\vartheta}
\end{aligned}
$$




$$
\geq \frac{1}{2}\left(1-\frac{\varepsilon\|\beta\|_{L^{\infty}(\Omega)}}{\hat{\lambda}_{1}^{+}(\beta)}\right)\|u\|^{2}-c_{3}\|u\|^{\vartheta}
$$

for some $c_{3}>0$ (see (4) and (15)).

Choosing $\varepsilon \in\left(0, \hat{\lambda}_{1}^{+}(\beta) /\|\beta\|_{L^{\infty}(\Omega)}\right)$, we obtain that

$$
\varphi_{+}(u) \geq \frac{c_{4}}{2}\|u\|^{2}-c_{3}\|u\|^{\vartheta} \quad \text { with } c_{4}=c_{4}(\varepsilon)>0 .
$$

Because $\vartheta>2$, from (16) we see that, for $\rho_{+} \in(0,1)$ small, we have

$$
\varphi_{+}(u) \geq \hat{m}_{+}>0 \quad \text { for all } u \in H_{0}^{1}(\Omega) \text {, with }\|u\|=\rho_{+} .
$$

A similar proof holds for the functional $\varphi_{-}$.

Recall that $\tilde{u}_{1}\left(\Omega_{+}\right) \in \operatorname{int} C_{+}\left(\Omega_{+}\right)$is the principal eigenfunction of $-\Delta$ in $H_{0}^{1}\left(\Omega_{+}\right)$ with weight $\beta^{+} \in L^{\infty}(\Omega)_{+}$. We extend $\tilde{u}_{1}$ to all of $\bar{\Omega}$ by setting $\tilde{u}_{1}$ to be equal to 0 on $\bar{\Omega} \backslash \Omega_{+}$. We denote this extension by $\bar{u}_{1}$. Evidently $\bar{u}_{1} \in C(\bar{\Omega}) \cap H_{0}^{1}(\Omega)$.

\section{PROPOSITION 6}

If hypotheses $H(\beta)$ and $H(f)$ hold, then $\lim \sup _{t \rightarrow \pm \infty} \varphi_{ \pm}\left(t \bar{u}_{1}\right)<0$.

\section{Proof}

For $t>0$, we have that

$$
\begin{aligned}
& \varphi_{+}\left(t \bar{u}_{1}\right)=\frac{t^{2}}{2}\left\|D \bar{u}_{1}\right\|_{L^{2}\left(\Omega, \mathbb{R}^{N}\right)}^{2}-\int_{\Omega} \beta(z) F_{+}\left(t \bar{u}_{1}\right) d z \\
& =\frac{t^{2}}{2}\left\|\bar{u}_{1}\right\|^{2}-\int_{\Omega} \beta^{+}(z) F\left(t \bar{u}_{1}\right) d z \quad\left(\text { note that } \bar{u}_{1} \geq 0\right) \\
& \Rightarrow \quad \frac{\varphi_{+}\left(t \bar{u}_{1}\right)}{t^{2}}=\frac{1}{2}\left\|\bar{u}_{1}\right\|^{2}-\int_{\Omega} \beta^{+}(z) \frac{F\left(t \bar{u}_{1}\right)}{t^{2}} d z \\
& \Rightarrow \quad \limsup _{t \rightarrow+\infty} \frac{\varphi_{+}\left(t \bar{u}_{1}\right)}{t^{2}} \\
& \leq \frac{1}{2}\left\|\bar{u}_{1}\right\|^{2}-\int_{\Omega} \beta^{+}(z) \liminf _{t \rightarrow+\infty} \frac{F\left(t \bar{u}_{1}\right)}{t^{2}} d z \quad \text { (by Fatou's lemma) } \\
& \leq \frac{1}{2}\left\|\bar{u}_{1}\right\|^{2}-\frac{\eta}{2} \int_{\Omega} \beta^{+}(z) \bar{u}_{1}^{2} d z \quad \text { with } \eta \in\left(\hat{\lambda}_{m}^{+}(\beta), \hat{\lambda}_{m+1}^{+}(\beta)\right) \\
& \text { (see hypothesis } H(f)(\mathrm{ii}) \text { ) } \\
& =\frac{1}{2}\left\|D \tilde{u}_{1}\right\|_{L^{2}\left(\Omega_{+}, \mathbb{R}^{N}\right)}^{2}-\frac{\eta}{2} \int_{\Omega_{+}} \beta^{+}(z) \tilde{u}_{1}^{2} d z \quad\left(\text { recall the definition of } \bar{u}_{1}\right) \\
& \leq \frac{1}{2}\left[1-\frac{\eta}{\hat{\lambda}_{1}^{\Omega_{+}}\left(\beta^{+}\right)}\right]\left\|\tilde{u}_{1}\right\|_{H_{0}^{1}\left(\Omega_{+}\right)}^{2} \quad(\text { see }(4)) \\
& <0 \quad(\text { see } H(f)(i i)) \text {. }
\end{aligned}
$$

A similar proof holds for the functional $\varphi_{-}$. 
Now we are ready to produce constant-sign solutions.

PROPOSITION 7

If hypotheses $H(\beta)$ and $H(f)$ hold, then problem (1) has at least two nontrivial constant-sign solutions

$$
u_{0} \in \operatorname{int} C_{+}(\bar{\Omega}) \quad \text { and } \quad v_{0} \in-\operatorname{int} C_{+}(\bar{\Omega}) .
$$

Proof

By virtue of Propositions 3, 5, and 6, we can apply Theorem 1 (the mountain pass theorem) and obtain $u_{0} \in H_{0}^{1}(\Omega)$ such that

$$
\varphi_{+}^{\prime}\left(u_{0}\right)=0 \quad \text { and } \quad \varphi_{+}(0)=0<\hat{m}_{+} \leq \varphi_{+}\left(u_{0}\right) .
$$

From (17) it is clear that $u_{0} \neq 0$. We have

$$
A\left(u_{0}\right)=\beta(z) N_{f_{+}}\left(u_{0}\right) .
$$

On (18) we act with $-u_{0}^{-} \in H_{0}^{1}(\Omega)$ and obtain

$$
\left\|u_{0}^{-}\right\|^{2}=0 ; \quad \text { hence } u_{0} \geq 0, u_{0} \neq 0 \text {. }
$$

Then (18) becomes

$$
\begin{aligned}
& A\left(u_{0}\right)=\beta(z) N_{f}\left(u_{0}\right) \\
& \quad \Rightarrow \quad-\Delta u_{0}(z)=\beta(z) u_{0}(z) \quad \text { a.e. in } \Omega,\left.\quad u_{0}\right|_{\partial \Omega}=0 .
\end{aligned}
$$

Standard regularity theory implies that $u_{0} \in C_{+}(\bar{\Omega}) \backslash\{0\}$. Let $\rho=\left\|u_{0}\right\|_{\infty}$. Hypotheses $H(\beta), H(f)(\mathrm{i})$, and $H(f)\left(\right.$ iii) imply that we can find $\xi_{\rho}>0$ such that

$$
\beta(z) f(x)+\xi_{\rho} x \geq 0 \quad \text { for a.a. } z \in \Omega, \text { for all } 0 \leq x \leq \rho .
$$

So, we have

$$
\begin{aligned}
& -\Delta u_{0}(z)+\xi_{\rho} u_{0}(z)=\beta(z) f\left(u_{0}(z)\right)+\xi_{\rho} u_{0}(z) \geq 0 \quad \text { a.e. in } \Omega \\
& \Rightarrow \quad \Delta u_{0}(z) \leq \xi_{\rho} u_{0}(z) \quad \text { a.e. in } \Omega \\
& \Rightarrow \quad u_{0} \in \operatorname{int} C_{+}(\bar{\Omega}) \quad \text { (by the maximum principle; see [9, p. 738]). }
\end{aligned}
$$

Similarly, working this time with the functional $\varphi_{-}$, we obtain another nontrivial constant-sign solution $v_{0} \in-\operatorname{int} C_{+}(\bar{\Omega})$.

Next we will produce a third nontrivial solution for problem (1). To do this, we use tools from Morse theory (critical groups). So, we first compute the critical groups of the energy functional $\varphi$ at infinity. This particular computation will be based on Proposition 2.

\section{PROPOSITION 8}

If hypotheses $H(\beta)$ and $H(f)$ hold, then $C_{k}(\tau, \infty)=\delta_{k, d_{m}} \mathbb{Z}$ for all $k \geq 0$ with some $d_{m} \geq 2$. 
Proof

Let $\hat{\eta} \in\left(\hat{\lambda}_{m}^{+}(\beta), \hat{\lambda}_{m+1}^{+}(\beta)\right)$, and consider the $C^{2}$-functional $\tau: H_{0}^{1}(\Omega) \rightarrow \mathbb{R}$ defined by

$$
\tau(u)=\frac{1}{2}\|D u\|_{2}^{2}-\frac{\hat{\eta}}{2} \int_{\Omega} \beta(z) u^{2} d z \quad \text { for all } u \in H_{0}^{1}(\Omega) .
$$

We consider the homotopy $h(t, u)=h_{t}(u)$ defined by

$$
h_{t}(u)=(1-t) \varphi(u)+t \tau(u) \text { for all }(t, u) \in[0,1] \times H_{0}^{1}(\Omega) .
$$

\section{CLAIM}

There exist $\mu \in \mathbb{R}$ and $\delta>0$ such that

$$
h_{t}(u) \leq \mu \quad \Rightarrow \quad(1+\|u\|)\left\|\left(h_{t}\right)^{\prime}(u)\right\|_{*}^{2} \geq \delta\|u\|^{2} \quad \text { for all } t \in[0,1] .
$$

We argue by contradiction. So, suppose that the claim is not true. Since the homotopy $(t, u) \mapsto h_{t}(u)$ maps bounded sets to bounded sets, we can find $\left\{t_{n}\right\}_{n \geq 1} \subseteq$ $[0,1]$ and $\left\{u_{n}\right\}_{n \geq 1} \subseteq H_{0}^{1}(\Omega)$ such that

$$
\left\{\begin{array}{l}
t_{n} \rightarrow t, \quad\left\|u_{n}\right\| \rightarrow \infty, \quad h_{t_{n}}\left(u_{n}\right) \rightarrow-\infty \quad \text { as } n \rightarrow \infty \quad \text { and } \\
\left|\left\langle\left(h_{t_{n}}\right)^{\prime}\left(u_{n}\right), v\right\rangle\right| \leq \frac{\|v\|}{n\left(1+\left\|u_{n}\right\|\right)}\left\|u_{n}\right\|^{2} \quad \text { for all } v \in H_{0}^{1}(\Omega), \text { for all } n \geq 1 .
\end{array}\right.
$$

From (19), we have that

$$
\begin{aligned}
& \left|\left\langle A\left(u_{n}\right), v\right\rangle-\left(1-t_{n}\right) \int_{\Omega} \beta(z) f\left(u_{n}\right) v d z-t_{n} \hat{\eta} \int_{\Omega} \beta(z) u_{n} v d z\right| \\
& \quad \leq \frac{\|v\|}{n\left(1+\left\|u_{n}\right\|\right)}\left\|u_{n}\right\|^{2} \quad \text { for all } n \geq 1 .
\end{aligned}
$$

Let $y_{n}=u_{n} /\left\|u_{n}\right\|, n \geq 1$. Then $\left\|y_{n}\right\|=1$ for all $n \geq 1$, and so we may assume that

$$
y_{n} \stackrel{w}{\rightarrow} y \quad \text { in } H_{0}^{1}(\Omega) \quad \text { and } \quad y_{n} \rightarrow y \quad \text { in } L^{2}(\Omega) .
$$

From (20) we obtain that

$$
\begin{aligned}
& \left|\left\langle A\left(y_{n}\right), v\right\rangle-\left(1-t_{n}\right) \int_{\Omega} \beta(z) \frac{f\left(u_{n}\right)}{\left\|u_{n}\right\|} v d z-t_{n} \hat{\eta} \int_{\Omega} \beta(z) y_{n} v d z\right| \\
& \quad \leq \frac{\|v\|}{n} \text { for all } n \geq 1 .
\end{aligned}
$$

Recall that $\left\{\beta N_{f}\left(u_{n}\right) /\left\|u_{n}\right\|\right\}_{n \geq 1} \subseteq L^{2}(\Omega)$ is bounded. So, if in (22) we choose $v=y_{n}-y \in H_{0}^{1}(\Omega)$ and pass to the limit as $n \rightarrow \infty$, then we obtain that

$$
\begin{aligned}
& \lim _{n \rightarrow \infty}\left\langle A\left(y_{n}\right), y_{n}-y\right\rangle=0 \\
& (23) \quad \Rightarrow \quad y_{n} \rightarrow y \quad \text { in } H_{0}^{1}(\Omega) \text { (as before via the Kadec-Klee property) } \\
& (24) \quad \Rightarrow \quad\|y\|=1 .
\end{aligned}
$$


Moreover, we know that, at least for a subsequence, we have

$$
\begin{aligned}
& \beta \frac{N_{f}\left(u_{n}\right)}{\left\|u_{n}\right\|} \stackrel{w}{\rightarrow} \eta \beta y \\
& \left.\quad \text { in } L^{2}(\Omega) \text { with } \eta \in\left(\hat{\lambda}_{m}^{+}(\beta), \hat{\lambda}_{m+1}^{+}(\beta)\right) \text { (see hypothesis } H(f)(\mathrm{ii})\right) .
\end{aligned}
$$

So, if in (22) we pass to the limit as $n \rightarrow \infty$ and use (23) and (25), then

$$
\begin{aligned}
\langle A(y), v\rangle=\eta_{t} \int_{\Omega} \beta(z) y v d z \quad \text { for all } v \in H_{0}^{1}(\Omega) \text { with } \eta_{t}=(1-t) \eta+t \hat{\eta} \\
\Rightarrow \quad A(y)=\eta_{t} \beta(z) y \\
(26) \quad \Rightarrow \quad-\Delta y(z)=\eta_{t} \beta(z) y(z) \quad \text { a.e. in } \Omega,\left.\quad y\right|_{\partial \Omega}=0 .
\end{aligned}
$$

Note that $\eta_{t} \in\left(\hat{\lambda}_{m}^{+}(\beta), \hat{\lambda}_{m+1}^{+}(\beta)\right)$. Then from (26) it follows that $y=0$, which contradicts (24). This proves the claim.

From Proposition 4 we know that $\varphi$ satisfies the $C$-condition. Similarly, since $\hat{\eta} \in\left(\hat{\lambda}_{m}^{+}(\beta), \hat{\lambda}_{m+1}^{+}(\beta)\right)$, it is easily seen that $\tau$ also satisfies the $C$-condition.

So, we can apply Proposition 2 and infer that

$$
C_{k}(\varphi, \infty)=C_{k}(\tau, \infty) \text { for all } k \geq 0 .
$$

The fact that $\hat{\eta} \in\left(\hat{\lambda}_{m}^{+}(\beta), \hat{\lambda}_{m+1}^{+}(\beta)\right)$ implies that $K_{\tau}=\{0\}$. Therefore

$$
C_{k}(\tau, \infty)=C_{k}(\tau, 0) \text { for all } k \geq 0 .
$$

Moreover, $u=0$ is a nondegenerate critical point of $\tau$ (i.e., $\tau^{\prime \prime}(0)$ is invertible), and from the minimax characterization of the eigenvalues $\left\{\hat{\lambda}_{k}^{+}(\beta)\right\}_{k \geq 1}$ (see Gasinski and Papageorgiou [9, p. 714]), we see that the Morse index of $\tau$ at $u=0$ is $d_{m} \geq 2$. So, we have that

$$
\begin{aligned}
& C_{k}(\tau, 0)=\delta_{k, d_{m}} \mathbb{Z} \quad \text { for all } k \geq 0 \\
& \quad \Rightarrow \quad C_{k}(\tau, \infty)=\delta_{k, d_{m}} \mathbb{Z} \quad \text { for all } k \geq 0 \text { with } d_{m} \geq 2(\text { see }(27),(28)) .
\end{aligned}
$$

This completes the proof.

Now we can produce the third nontrivial solution.

\section{PROPOSITION 9}

If hypotheses $H(\beta)$ and $H(f)$ hold, then problem (1) has a third nontrivial solution $y_{0} \in C_{0}^{1}(\bar{\Omega})$.

Proof

From Proposition 7 we already have two nontrivial solutions of constant sign

$$
u_{0} \in \operatorname{int} C_{+}(\bar{\Omega}) \quad \text { and } \quad v_{0} \in-\operatorname{int} C_{+}(\bar{\Omega}) .
$$

From the proof of that proposition, we know that

(i) $u_{0}$ is a critical point of $\varphi_{+}$of mountain pass type;

(ii) $v_{0}$ is a critical point of $\varphi_{-}$of mountain pass type. 
Hence we have that

$$
C_{1}\left(\varphi_{+}, u_{0}\right) \neq 0 \quad \text { and } \quad C_{1}\left(\varphi_{-}, v_{0}\right) \neq 0 .
$$

Note that $\left.\varphi_{+}\right|_{C_{+}}=\left.\varphi\right|_{C_{+}}$and $\left.\varphi_{-}\right|_{-C_{+}}=\left.\varphi\right|_{-C_{+}}$. Since $u_{0} \in \operatorname{int} C_{+}(\bar{\Omega})$ and $v_{0} \in$ $-\operatorname{int} C_{+}(\bar{\Omega})$ it follows that

$$
\begin{cases}C_{k}\left(\left.\varphi_{+}\right|_{C_{0}^{1}(\bar{\Omega})}, u_{0}\right)=C_{k}\left(\left.\varphi\right|_{C_{0}^{1}(\bar{\Omega})}, u_{0}\right) & \text { for all } k \geq 0 \\ C_{k}\left(\left.\varphi_{-}\right|_{C_{0}^{1}(\bar{\Omega})}, v_{0}\right)=C_{k}\left(\left.\varphi\right|_{C_{0}^{1}(\bar{\Omega})}, v_{0}\right) & \text { for all } k \geq 0\end{cases}
$$

Since $C_{0}^{1}(\bar{\Omega})$ is dense in $H_{0}^{1}(\bar{\Omega})$, from Palais [12] and (30), we have that

$$
\begin{gathered}
C_{k}\left(\varphi_{+}, u_{0}\right)=C_{k}\left(\varphi, u_{0}\right) \quad \text { and } \quad C_{k}\left(\varphi_{-}, v_{0}\right)=C_{k}\left(\varphi, v_{0}\right) \quad \text { for all } k \geq 0 \\
\Rightarrow \quad C_{1}\left(\varphi, u_{0}\right) \neq 0 \quad \text { and } \quad C_{1}\left(\varphi, v_{0}\right) \neq 0 \quad(\text { see }(29)) \\
\Rightarrow \quad C_{k}\left(\varphi, u_{0}\right)=C_{k}\left(\varphi, v_{0}\right)=\delta_{k, 1} \mathbb{Z} \quad \text { for all } k \geq 0 \\
\text { (see Bartsch }[6, \text { Proposition 2.5]). }
\end{gathered}
$$

Also, using (15) we have that

$$
\begin{aligned}
\varphi(u) & \geq c_{5}\|u\|^{2}-c_{6}\|u\|^{\vartheta} \quad \text { for all } u \in H_{0}^{1}(\Omega) \text { and some } c_{5}, c_{6}>0(\text { recall } \vartheta>2) \\
\Rightarrow & u=0 \quad \text { is a local minimizer of } \varphi \\
(32) & \Rightarrow \quad C_{k}(\varphi, 0)=\delta_{k, 0} \mathbb{Z} \quad \text { for all } k \geq 0 .
\end{aligned}
$$

From Proposition 8 we know that $C_{k}(\varphi, \infty)=\delta_{k, d_{m}} \mathbb{Z}$ for all $k \geq 0$. This means that there exists $y_{0} \in K_{\varphi}$ such that

$$
C_{d_{m}}\left(\varphi, y_{0}\right) \neq 0 \text {. }
$$

Since $d_{m} \geq 2$, comparing (32) with (30) and (31), we infer that

$$
\begin{aligned}
y_{0} & \notin\left\{0, u_{0}, v_{0}\right\} \\
& \left.\Rightarrow y_{0} \text { is a third nontrivial solution of (1) (since } y_{0} \in K_{\varphi}\right) .
\end{aligned}
$$

Standard regularity theory implies that $y_{0} \in C_{0}^{1}(\bar{\Omega})$.

So, summarizing the situation, we can state the following multiplicity theorem for problem (1).

\section{THEOREM 10}

If hypotheses $H(\beta)$ and $H(f)$ hold, then problem (1) has at least three nontrivial solutions

$$
u_{0} \in \operatorname{int} C_{+}(\bar{\Omega}), \quad v_{0} \in-\operatorname{int} C_{+}(\bar{\Omega}), \quad \text { and } \quad y_{0} \in C_{0}^{1}(\bar{\Omega}) .
$$

Acknowledgment. Rădulescu's work supported by grant CNCS-PCCA-23/2014. 


\section{References}

[1] S. Alama and G. Tarantello, On semilinear elliptic equations with indefinite nonlinearities, Calc. Var. Partial Differential Equations 1 (1993), 439-475. MR 1383913. DOI 10.1007/BF01206962.

[2] _ Elliptic problems with nonlinearities indefinite in sign, J. Funct. Anal. 141 (1996), 159-215. MR 1414377. DOI 10.1006/jfan.1996.0125.

[3] H. Amann and E. Zehnder, Nontrivial solutions for a class of nonresonance problems and applications to nonlinear differential equations, Ann. Sc. Norm. Sup. Pisa Cl. Sci. (4) 7 (1980), 539-603. MR 0600524.

[4] A. Ambrosetti and A. Malchiodi, Nonlinear Analysis and Semilinear Elliptic Problems, Cambridge Stud. Adv. Math. 104, Cambridge Univ. Press, Cambridge, 2007. MR 2292344. DOI 10.1017/CBO9780511618260.

[5] A. Ambrosetti and P. Rabinowitz, Dual variational methods in critical point theory and applications, J. Funct. Anal. 14 (1973), 349-381. MR 0370183.

[6] T. Bartsch, Critical point theory on partially ordered Hilbert spaces, J. Funct. Anal. 186 (2001), 117-152. MR 1863294. DOI 10.1006/jfan.2001.3789.

[7] H. Berestycki, I. Capuzzo-Dolcetta, and L. Nirenberg, Superlinear indefinite elliptic problems and nonlinear Liouville theorems, Topol. Methods Nonlinear Anal. 4 (1994), 59-78. MR 1321809.

[8] K.-C. Chang and M.-Y. Jiang, Dirichlet problem with indefinite nonlinearities, Calc. Var. Partial Differential Equations 20 (2004), 257-282. MR 2062944. DOI 10.1007/s00526-003-0236-7.

[9] L. Gasiński and N. S. Papageorgiou, Nonlinear Analysis, Ser. Math. Anal. Appl. 9, Chapman \& Hall/CRC, Boca Raton, Fla., 2006. MR 2168068.

[10] S. Hu and N. S. Papageorgiou, Three nontrivial solutions for noncoercive asymptotically linear elliptic problems, Houston J. Math. 37 (2011), 559-576. MR 2794563.

[11] T. Ouyang, On the positive solutions of semilinear equations $\Delta u+\lambda u-h u^{p}=0$ on compact manifolds, Trans. Amer. Math. Soc. 331 (1992), 503-527. MR 1055810. DOI 10.2307/2154124.

[12] R. S. Palais, Homotopy theory of infinite dimensional manifolds, Topology 5 (1966), 1-16. MR 0189028.

[13] N. S. Papageorgiou and V. Rădulescu, Resonant $(p, 2)$-equations with asymmetric reaction, Anal. Appl., published electronically 21 April 2014. DOI 10.1142/S0219530514500134.

[14] N. S. Papageorgiou and G. Smyrlis, Nonlinear elliptic equations with an asymptotically linear reaction term, Nonlinear Anal. 71 (2009), 3129-3151. MR 2532837. DOI 10.1016/j.na.2009.01.224.

Papageorgiou: Department of Mathematics, National Technical University, Zografou Campus, Athens 15780, Greece; npapg@math.ntua.gr 
Rădulescu: Department of Mathematics, Faculty of Science, King Abdulaziz University, Jeddah, Saudi Arabia; and Institute of Mathematics "Simion Stoilow" of the Romanian Academy, Bucharest, Romania; vicentiu.radulescu@math.cnrs.fr 